\title{
Human organ repair centers: Fact or fiction?
}

\section{Shaf Keshavjee, MD, MSc}

\begin{abstract}
Feature Editor Note-Although lung transplantation continues to evolve with the refinement of surgical techniques and improvements in organ preservation, there continues to be a critical shortage of donor lungs available, and a substantial number of donor lungs are discarded due to questionable quality. The University of Toronto group has published extensively on the use of ex vivo lung perfusion (EVLP) to evaluate marginal donor lungs, demonstrating that many of these lungs can be successfully transplanted. However, the necessary equipment, logistical support, and expertise are not readily available at most transplant centers. Dr Keshavjee is an authoritative expert in this area, and this Invited Expert Opinion article provides an excellent overview of the development of EVLP organ repair centers, which would not only make EVLP more widely available but offer the potential for incorporating precision diagnostics into organ management and repairing damaged donor lungs in the future. The article draws parallels between the development of centralized, standardized blood banks and the potential for regional EVLP organ repair centers to become part of an organ-management ecosystem. The establishment of 2 lung bioengineering centers has brought the concept of EVLP organ repair centers closer to fact than fiction and has the potential to transform lung transplantation by increasing donor lung use and possibly improving long-term outcomes.
\end{abstract}

\section{Jules Lin, MD}

Organ transplantation therapy has continued to evolve and enjoy increased success. The development of organ transplantation was made possible by the development of surgical techniques and organ-preservation strategies. Preservation started with recovery of an organ from a person who had died and essentially aimed at preserving the organ in the state in which it was found. We traditionally would rush through retrieval, transport, and implantation with the hope that cold flush preservation would preserve the organ, hopefully preventing significant degradation so we

From the Toronto Lung Transplant Program, Sprott Department of Surgery, University Health Network, and Division of Thoracic Surgery, University of Toronto, Toronto, Canada

Received for publication May 6, 2020; revisions received May 6, 2020; accepted for publication May 6, 2020; available ahead of print Sept 9, 2020.

Address for reprints: Shaf Keshavjee, MD, MSc, Toronto General Hospital, 200 Elizabeth St, 9N946, Toronto, Ontario MSG 2C4, Canada (E-mail: Shaf. keshavjee@uhn.ca).

JTCVS Open 2020;3:164-8

2666-2736

Copyright $(2020$ The Authors. Published by Elsevier Inc. on behalf of The American Association for Thoracic Surgery. This is an open access article under the CC BY-NCND license (http://creativecommons.org/licenses/by-nc-nd/4.0/).

https://doi.org/10.1016/j.xjon.2020.05.001

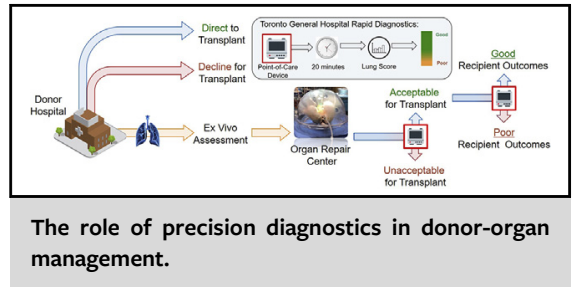

\begin{abstract}
CENTRAL MESSAGE
The transplant ecosystem is evolving with better management strategies-leading to a precision medicine approach to preservation, transportation, and management of organs optimized for transplantation.
\end{abstract}

See Commentaries on pages 169,171 , and 173.

could implant an organ in as close as possible state to that in which we found it. This has served us for well over 50 years. The question is, how do we take transplantation to the next level? Can we develop better systems for organ management and delivery of transplant care? Can we aspire to make organs better-can we make a lung or a heart that is better than the way we found it in the person who died? Can we engineer and pre-prepare organs that look like "self" so that they won't be rejected? Can we make an organ that will outlive the recipient in which it is placed?

We set out set out some years ago on a quest to do just that - to develop strategies to repair donor lungs, ultimately to engineer lungs that are pre-prepared for transplantation. Given the logistical challenges of working on organs in the multiorgan donor, we set out to develop an ex vivo support system for lungs, such that you could maintain lungs outside of the body at normal body temperature. This allows one to work on the organ-apply precision diagnostics and therapeutics of modern medicine, just as we do in patients. We put together all that we had learned over many years about ideal protective perfusion and ventilation strategies for the lung, creating a system that could maintain the lung outside the body for 12 hours and not inflict significant damage. The concept is not a stress test but a protective support system for the organ. ${ }^{1}$ We translated this to clinical practice in the Toronto Ex Vivo Lung Perfusion (EVLP) 
System (Figure 1), demonstrating that we could safely transplant lungs we would not have used before. ${ }^{2}$ This has transformed the approach to organ preservation and changed the field of transplantation. A whole new industry is growing around ex vivo organ perfusion systems and the development of techniques to support and treat not just lungs, but all organs outside the body.

Is normothermia necessary? The fundamental premise of hypothermic flush preservation that has made the miracle of transplantation possible is that cold temperature decreases the metabolic rate of the organ. Cold slows down the consumption of metabolic substrate, the production of wastes, and essentially slows down the dying process. However, cold also slows down opportunities to diagnose, repair, and regenerate the organ. Thus, although cold is needed for certain phases of the transplant process, normothermia is also necessary if we are to aspire to repair and regenerate organs. Now with EVLP, for the first time, we have the opportunity to manipulate preservation temperature as needed. This provides time to accurately assess and diagnose the organ, which has significantly improved use and the opportunity to treat, recover, and repair organs in a precision medicine approach, where we can confirm the results such that we can more confidently transplant an organ with a predictable outcome. This will ultimately

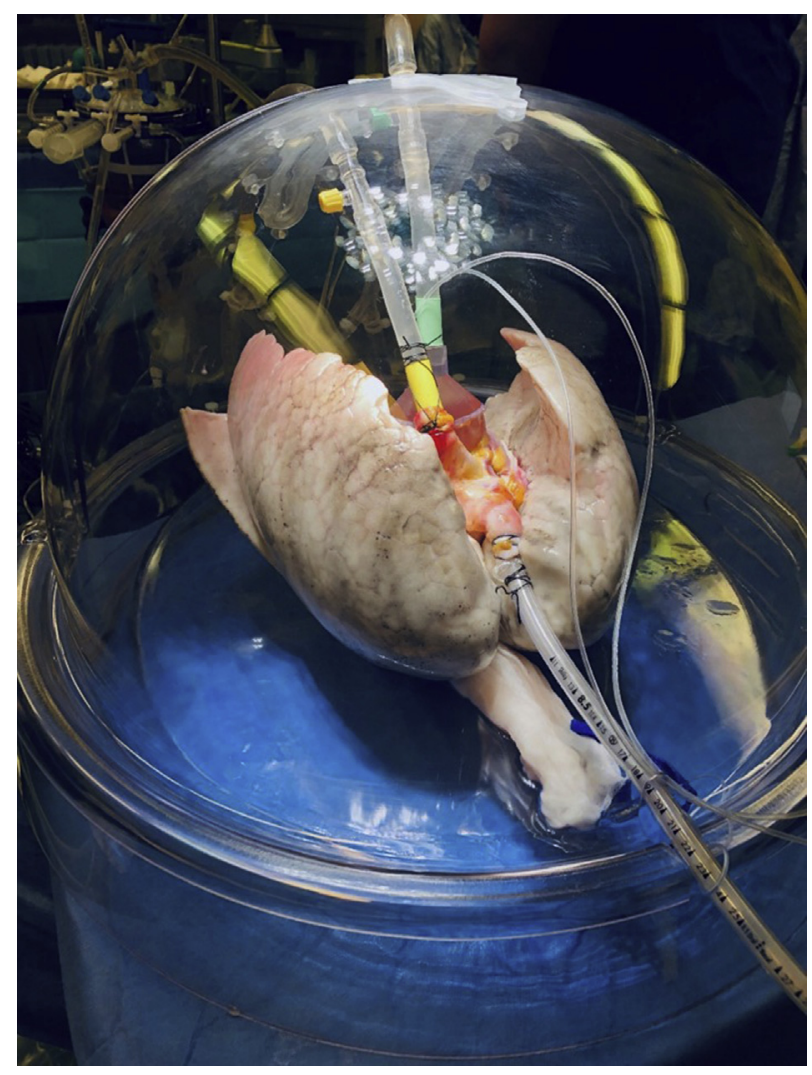

FIGURE 1. The Toronto Ex Vivo Lung Perfusion system. improve the safety and long-term outcomes of transplantation.

We first introduced EVLP clinically at Toronto General Hospital (TGH) in 2008 and since then have performed more than 600 cases. As mentioned, we demonstrated that we could successfully transplant extended donor lungs that we would have otherwise declined for transplantation. Now, with more than a decade of EVLP experience, we have shown that these lungs have an equivalently good long-term outcome to standard donor lungs used in our program. ${ }^{3}$ The use of the Toronto EVLP System has essentially led to a doubling of the number of lung transplants performed per year at our center in the last 5 years, where we now perform more than 200 lung transplants per year.

To take this concept to the next level, we need to exploit the real opportunity that EVLP provides, the opportunity to improve donor lungs - to treat infections, perform gene modifications to manipulate the immune response, use stem cells to repair and regenerate the organ, and use other pharmacologic and biologic therapies to improve organs and truly engineer organs that are superior to the condition in which we found them. One may think that this is taking an already-complex, challenging, and expensive process, that is, transplantation, and making it even more so. Historically, the field of transplantation has developed organically; the current state is highly inefficient and not conducive to the scaling of activity that will be required. We need to examine how we can modify and develop the transplant ecosystem to optimize the use of organs and resources to enhance access to life-saving organ transplantation.

We can take a lesson from the development of blood transfusion (Figure 2, A). The first blood transfusion described was from a healthy soldier in the field to a soldier that was hemorrhaging, to save his life. You can imagine with that practice that they saved quite a few lives, but they also likely killed a few people with blood transfusion reactions and infections. Therefore, we developed small "blood banks" in MASH units and in hospitals around the country, where typed units of blood were kept to be more readily available. This did a little better with rudimentary blood typing, but standardization of practice and quality was lacking. There was risk of error and there was no really efficient way to preserve and distribute this scarce resource. Thus, we went on to develop centralized, standardized collection centers, processing centers, and distribution centers. We now have standardized operating protocols with oversight of processing, quality, infection control, shelf life, inventory, and distribution tracking. Moreover, with advanced processing techniques, we have also optimized use of this scarce resource with the separation of blood into components so that a patient only receives the component they need (eg, red cells, platelets, cryoprecipitate, etc) and each unit of blood provides life-saving therapy for 

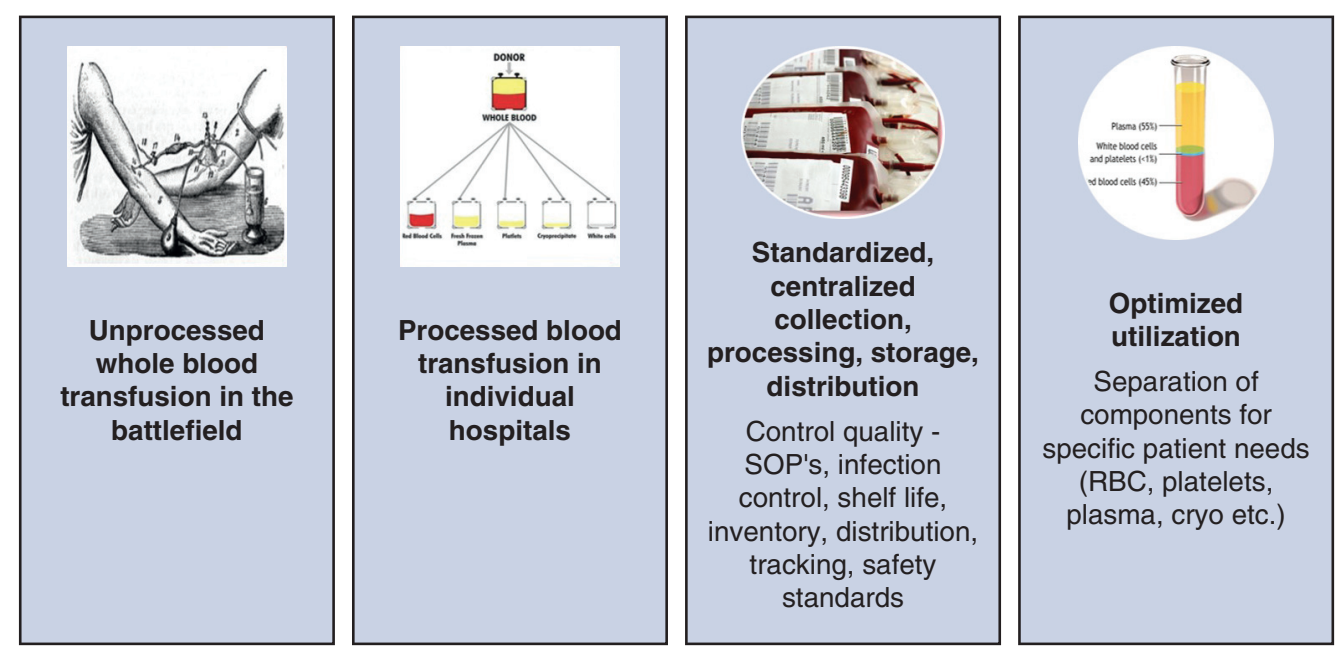

Scale up, Achieve Cost and Utilization Efficiencies

Management of Blood Products: The Evolutionary Path

A

The Future State of Organ Transplantation

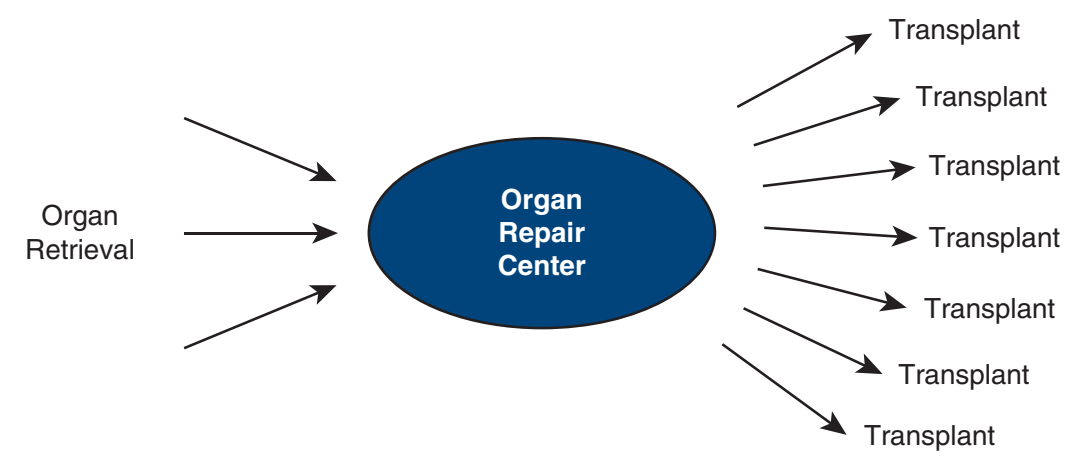

B

FIGURE 2. A, The evolution of blood transfusion provides a lesson to the field of organ transplantation. B, The future state of organ transplantation. Organs will be transported to an organ repair center, where they will be treated, optimized, and transported to the recipient site for transplantation. SOP, Standard operating protocol; $R B C$, red blood cell.

multiple patients. This has allowed us to scale up and achieve remarkable safety and access as well as cost and use efficiencies in transfusion practice.

We can apply these same concepts to the processing and management of organs for transplantation. Instead of having 5 Lear jets fly out every night, with 5 surgical teams to pick up 1 organ each and then fly back to 5 different hospitals, you will have organs being transported to an organ repair center, processed, optimized, and then transported to each patient (Figure 2, B). Furthermore, why would you need a whole jet to transport and organ that weighs less than $2 \mathrm{~kg}$ ? In fact, we are now developing Remote Piloted Aircraft Systems (RPAS, or drones) to deliver organs. This is the future of transplantation (Figure 3).

This may sound like science fiction but we, in fact, established the first Organ Repair Center in the world more than 10 years ago at TGH-essentially a mini-operating room to support and treat organs for transplant. We started with lung and then expanded to liver, kidney, and now heart ex vivo perfusion. We demonstrated the possibility of providing a service of lung repair for another center. In a compassionate case, we flew a lung from a remote donor hospital to Toronto for EVLP and then flew it to 


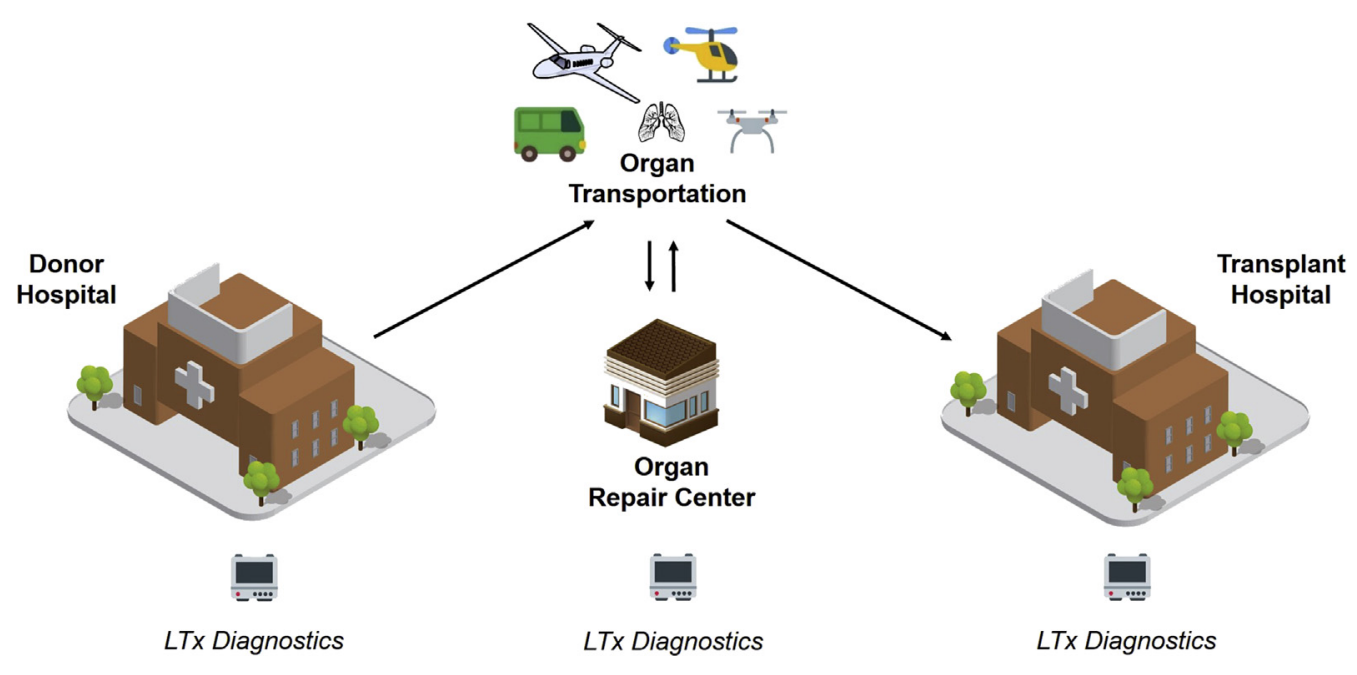

FIGURE 3. The future state of the organ-management ecosystem. LTx, Lung transplantation.

the recipient hospital where it was successfully transplanted-the first case demonstrating the "organ repair center" concept of remote organ perfusion treatment for transplantation. ${ }^{4}$

To expand this concept into the United States, in partnership with United Therapeutics, the subsidiary called Lung Bioengineering was founded. Lung Bioengineering 1 (LB1) is a 28,000-square foot facility in Silver Spring, Maryland, that is the world's first "lung hospital," designed to repair lungs for clinical transplantation. There are 6 procedure rooms that have the capacity to treat 1800 lungs a year in that facility alone. Organ perfusion specialists trained at TGH perform EVLP for transplant centers in the United States. There is complete data-monitoring and information feed where the transplant surgeon receives all the physiologic and biologic parameters as well as imaging by radiography or bronchoscopy remotely by data, audio, and video contact directly on a smartphone or tablet device. More than
100 lungs have been transplanted after EVLP at LB1 to date. Lung Bioengineering 2 (LB2) has recently opened in Jacksonville, Florida, to provide both redundancy and expansion of service. These 2 facilities alone have the capability to potentially cover EVLP services for the whole continent, as has been demonstrated by the Toronto Lung Transplant Program experience with remote organ retrieval and transplantation, opening the door to the possibility of semi-elective lung transplantation. ${ }^{5,6}$ The organ repair center concept is indeed a reality that has now moved into the phase of a commercial entity to provide the service of EVLP for transplant centers in the United States. The development of regional organ procurement centers ${ }^{7}$ is complementary and synergistic to the development of regional ex vivo organ procurement centers. In fact, optimization of donor organ use and outcomes will likely be achieved by a combination of both concepts.

A further requirement will be the development and application of rapid advanced diagnostics to the field of organ

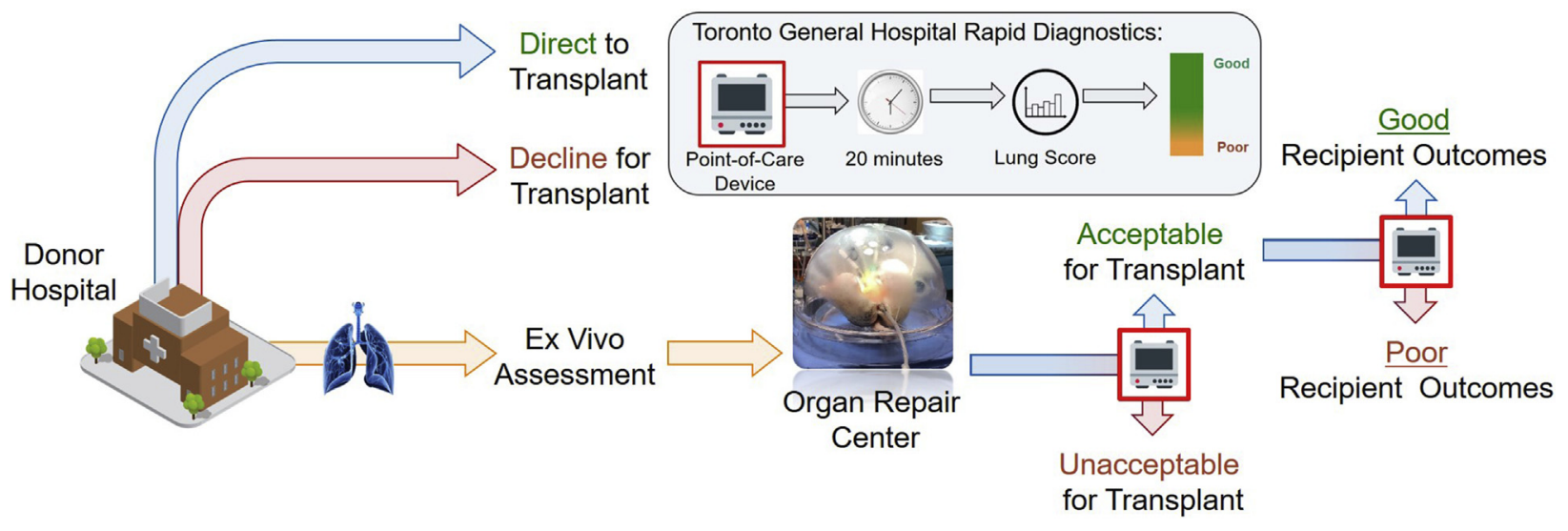

FIGURE 4. The role of precision diagnostics in donor-organ management. 
preservation and management (Figure 4). Currently, donor organ assessment is basically standard clinical assessment. Advanced rapid diagnostic profiling will be required to bring the precision medicine concept to the bedside of donor organ management. Is this lung okay to go straight to transplant, or do you need to repair it? If you do need to repair it, diagnose the problem, define your prescription of repair, repair it, confirm that your lung is repaired, and transport it to your recipient to implant an organ that is a known product with a predicable good outcome.

A whole industry is developing around machines to perfuse organs, diagnostics to improve precision in decision making, and service providers to manage the organs. Furthermore, we cannot continue to build whole operating rooms to manage one organ at a time. Devices are being developed to automate these complex processes for each organ (lung, liver, kidney, heart), so that an organ perfusion specialist can monitor and treat multiple organs at the same time. Important health economic analyses should include examination of the combined cost effectiveness of EVLP in the context of the value of increased availability of donor lungs-which will save more lives and increased access to transplantation, and shorten the duration of care required for advanced end stage lung disease patients, combined with the benefit gained from more efficient organ management and transportation and ultimately from transplantation of organs with better and more predictable improved short and long term outcomes.

The field of transplantation has changed. We have an unprecedented opportunity to use more organs and improve the outcomes of transplantation. The clinical translation of EVLP technology has demonstrated the way forward for the development of a redesigned transplant ecosystem. Organs will be retrieved from a donor by a regional surgical retrieval team (or in a donor-management center), transported by drone to an organ repair center, treated and optimized for transplantation, and transported to the recipient for elective transplantation. This is fact, not fiction: clinical organ repair centers are already up and running. Furthermore, the components are coming together to reengineer the transplant ecosystem to create a much more efficient, cost-effective, and sustainable delivery strategy for clinical organ transplantation.

\section{Conflict of Interest Statement}

Dr Keshavjee is Chief Scientific Officer and founding partner of Perfusix Canada Inc, Perfusix USA Inc, and XOR Labs Toronto; he is a consultant to Lung Bioengineering and United Therapeutics; and has received research support from XVIVO Perfusion, United Therapeutics, and CSL Behring.

The Journal policy requires editors and reviewers to disclose conflicts of interest and to decline handling or reviewing manuscripts for which they may have a conflict of interest. The editors and reviewers of this article have no conflicts of interest.

\section{References}

1. Cypel M, Yeung JC, Hirayama S, Rubacha M, Fischer S, Anraku M, et al. Technique for prolonged normothermic ex vivo lung perfusion. J Heart Lung Transplant. 2008;27:1319-25.

2. Cypel M, Yeung JC, Liu M, Anraku M, Chen F, Karolak W, et al. Normothermic ex vivo lung perfusion in clinical lung transplantation. N Engl J Med. 2011;364: 1431-40.

3. Divithotawela C, Cypel M, Martinu T, Singer LG, Juvet S, Binnie M, et al. Longterm outcomes of lung transplantation with ex-vivo lung perfusion: a single centre 10 year experience. JAMA Surg. 2019;154:1143-50.

4. Wigfield CH, Cypel M, Yeung J, Waddell T, Alex C, Johnson C, et al. Successful emergent lung transplantation after remote ex vivo perfusion optimization and transportation of donor lungs. Am J Transplant. 2012;12: 2838-44.

5. Yeung JC, Krueger T, Yasufuku K, de Perrot M, Pierre AF, Waddell TK, et al. Outcomes after transplantation of lungs preserved for more than $12 \mathrm{~h}$ : a retrospective study. Lancet Respir Med. 2017;5:119-24.

6. Cypel M, Yeung JC, Keshavjee S. Introducing the concept of semielective lung transplantation through the use of ex vivo lung perfusion. J Thorac Cardiovasc Surg. 2018;156:2350-2.

7. Chang SH, Kreisel D, Marklin GF, Cook L, Hachem R, Kozower BD, et al. Lung focused resuscitation at a specialized donor care facility improves lung procurement rates. Ann Thorac Surg. 2018;105:1531-6. 